\title{
Importance of CAD Tools and Methodologies in High Speed CPU Design
}

\author{
Haruyuki Tago $\dagger \quad$ Kazuhiro Hashimoto $\dagger$ Nobuyuki Ikumi $\dagger$ Masato Nagamatsu $\uparrow$ \\ Masakazu Suzuoki • Yasuyuki Yamamoto•
}

$\dagger$ System ULSI Engineering Lab. $\ddagger$ System LSI Design Division

Toshiba Corporation, Semicondoctor Company

580-1, Horikawa-cho, Saiwai-ku

Kawasaki, Japan 212-8520

Tel : +81-44-548-2521

Fax : +81-44-548-8324

e-mail : tago@sdel.toshiba.co.jp

\author{
- R\&D Division \\ Sony Computer Entertainment Inc. \\ 1-1, Akasaka 7-chome, Minato-ku \\ Tokyo, Japan 107-0052 \\ $+81-3-5413-8669$ \\ $+81-3-5413-8631$
}

\begin{abstract}
Design methodologies and CAD for "Emotion Engine" LSI are presented with emphasis on practical aspects of verification and timing closure. A combination of simulation, emulation and formal verification ensured the functional first silicon for system evaluation. In order to control wire delay in early design stage, floor-plan based synthesis and wire load estimation are adopted for quick timing closure.
\end{abstract}

\section{Introduction}

"Emotion Engine" ("EE") is a system LSI including a $300 \mathrm{MHz} 128$-bit 2-way superscalar RISC core, two Vector Units ("VU"s), Image Processing Unit ("IPU") for MPEG-2 stream decode, a 10-channel memory access (DMA) controller, two channel Rambus ${ }^{\circledR}$ memory controller (RAC) and other peripheral modules [1], [2]. 13.5M transistors are integrated on $15.02 \mathrm{~mm} \times 15.04 \mathrm{~mm}$ die with $0.25 \mathrm{um}$ device technology. The chip photo and the block diagram are shown in Fig1.and Fig.2, respectively. Not only the RISC core but also both VPU0 and VPU1 have own program codes. This complexity makes verification more difficult than a single processor chip. These three processors which are running at $300 \mathrm{MHz}$ synchronously, occupies as large as $128 \mathrm{~mm}^{2}$ on the die. So, a careful timing design and clock skew management are required. This paper focuses on verification and timing closure because they are most crucial in this development project.

\section{Verification Methodology}

EE integrates several processors, including the 128-bit RISC core, VPU0 and VPU1 and the IPU. Concurrent data transfers on a 128-bit wide on-chip bus happen among them either by program control or by a 10 channel DMA. In order to manage this complexity, three verification approaches are adopted. They are simulation, emulation and formal Verification. Overall Verification flow is shown in Fig.3.

\section{A. Simulation}

Simulation statistics is summarized in Table.I. RTL simulation speed for whole $\mathrm{EE}$ is about 15 cycles/second. This is acceptable for basic function test but too slow for complex and application-like test. For example, it would have taken 571 days for whole EE simulation (740Mcycles) if one UltraSPARC-II class workstation had been used.

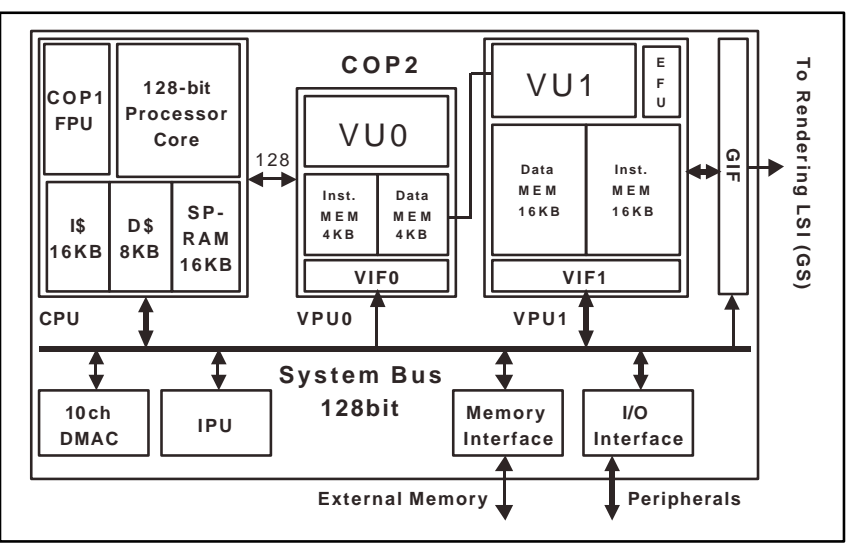

Fig. 2 Emotion Engine Block Diagram

TABLE I

RTL Simulation Cycles (unit: Mcycles)

\begin{tabular}{|c|c|c|c|}
\hline \multicolumn{4}{|l|}{ Major Block Level } \\
\hline & RISCcore & VU0 & VU1 \\
\hline Basic Function Test & 91.1 & 7.4 & 7.4 \\
\hline Random Program Test & 20000 & 23.4 & 216 \\
\hline
\end{tabular}

\begin{tabular}{|c|c|}
\hline Whole "EE" Level & "EE" \\
\hline \hline Basic Function Test & 121 \\
\hline Complex Case Test & 559 \\
\hline Application-like(but small) & 60 \\
\hline
\end{tabular}

\section{B. Emulation}

In order to speed verification process, a hardware emulation system is used. Linux OS is ported to the RISC 
core for verification purpose. For emulation of whole "EE", an external hardware board is designed and connected to the emulation system to verify the interface protocols. The emulation speed is $614 \mathrm{~K}$ cycles/second, roughly $40,000 \mathrm{X}$ faster than RTL simulation. Emulation speed is attractive but it often takes time to map EE gate netlist to the emulation system and difficult to debug.

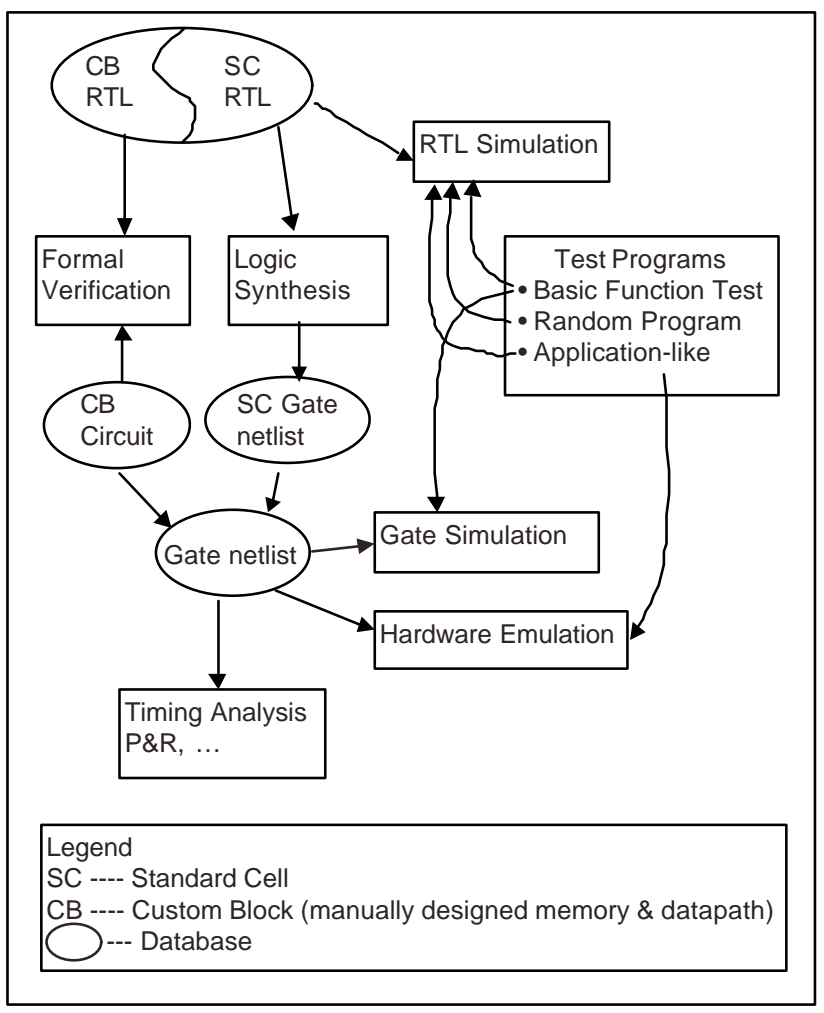

Fig. 3 Verification flow

\section{Formal verification}

Formal verification is applied to verify the equivalence of the different designs, including handcrafted custom block (CB) circuit vs. its RTL description.

\section{Timing Closure}

As design rule has been shrunk to 0.25-0.18 um level with higher clock frequency, controlling interconnect delay has become significant task in LSI design projects. We think the nature of this problem lies in the fact that floor-plan, RTL/gate structure and interconnection delay have become much more "closely-coupled" each other. For example, one of critical paths of the RISC core is so-called "Load Path" from the data cache memory to the integer register through the integer datapath. The designers' interests at early design stage would include:

- how much the path delay is (relative to other critical paths),

- how much timing would be improved if another floorplan is applied.

We advocate that the key is quick timing analysis and feedback allowing the designer try "what-if" analysis. Interactive improvements of floor-plan, RTL, synthesis script and $\mathrm{P} \& \mathrm{R}$ constraints are essential to solve timing problems throughout this design.

\section{A. Floor-plan based synthesis and interconnection estimation}

Design methodology of $300 \mathrm{MHz}$ VU(Vector Engine) ) is presented [3]. The key points are:

- careful functional design based on the VU architecture,

- consistent design hierarchy from RTL to Floor-plan,

- estimation of wire shape for pre-layout static timing analysis.

As a result, it takes only 15.4 hours from RTL to pre-layout timing analysis as far as the RTL or the floor-plan is not drastically changed. Reasonable estimation accuracy for early/middle design stage is obtained. It takes 85.2 hours to get post-layout timing analysis results with high accuracy. 15.4 hours allows the designers to improve RTL, floor-plan, $\mathrm{P} \& \mathrm{R}$ constraints, etc. every day, while 85.2 hours is close to every 4 days.

\section{B. Repeater insertion}

Even if most interconnections are managed well, long interconnections are inevitable. They are kind of the RISC stall signals and the bus signals used many locations over the chip. "RePertory", an automatic repeater insertion program, has been newly developed in this design project and is used effectively [4]. Repeater theory itself was established and not new. However, in order to apply actual LSI design, we think that some improvements are still necessary. RepPertory features include:

- Good controllability of the repeater location (topological and geometrical) and the number of repeaters for the optimum result,

- Preservation of the module boundary for enabling the following In-Place-Optimization (IPO),

- Nets for repeaters to be inserted are controllable (force or prohibit repeater insertion for each net).

\section{Clock skew management}

Clock skew must be minimized in order to improve the maximum clock rate, evade a race condition, and guarantee more timing margin for circuit designers. Many studies on automation of clock layout and synthesis have been reported and ASIC design flow can utilize the automated methodology effectively because of its standard cell (SC) based flexible layout. In contrast, almost all the clock network of EE is designed and drawn manually since a high percentage of the die area is occupied by custom blocks (CB's) and the floor plan is not flexible enough for the automated clock design. In those circumstances, an automated clock tuning method is developed to get accurate timing results promptly [5]. Less than $116 \mathrm{ps}$ overall clock skew has been achieved across $15.02 \times 15.04 \mathrm{~mm}$ die. 


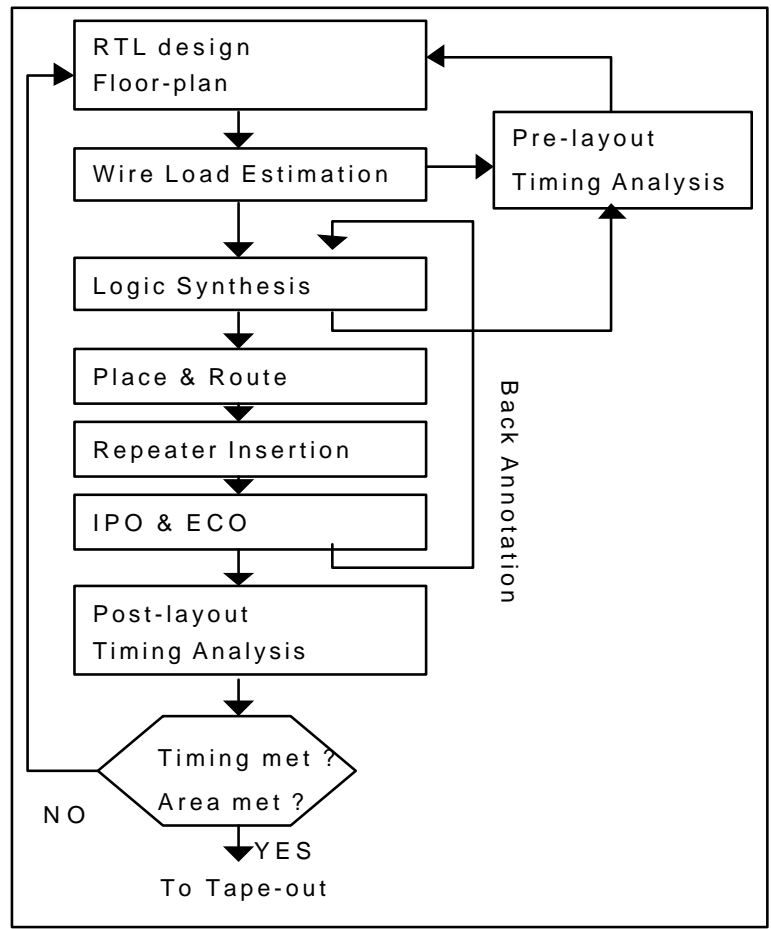

Fig. 3 Timing design flow

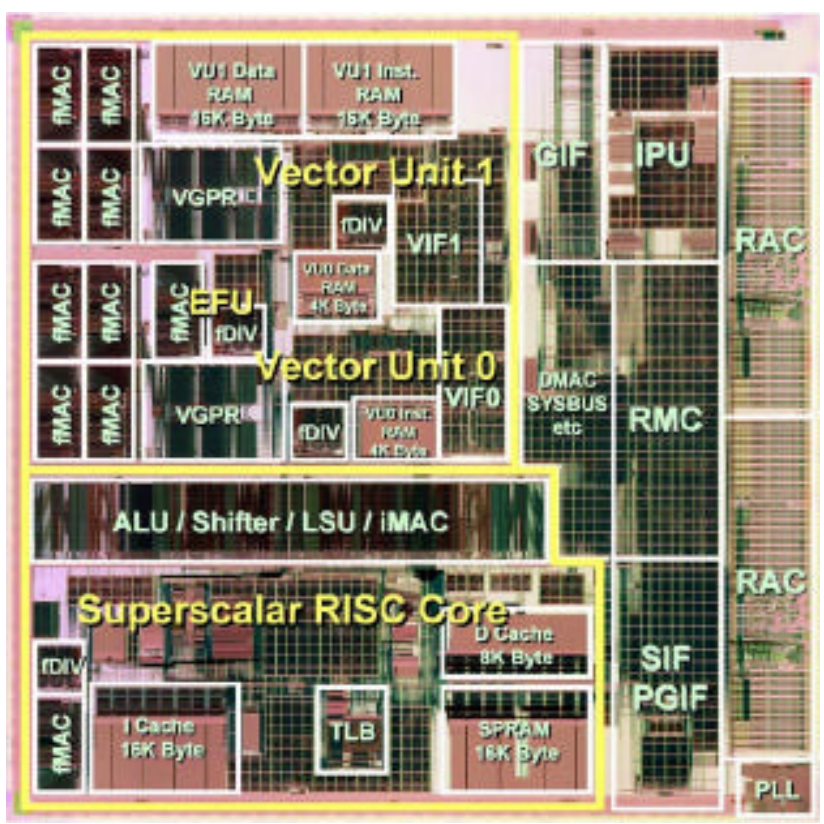

Fig.1 "Emotion Engine" Chip Photo

IV. Concluding Remarks

An integration trend for $\mathrm{PC}-\mathrm{CPU}$ and game-CPU is shown in Fig.4. Within several years, a game-CPU with nearly $100 \mathrm{M}$ transistors is expected to emerge! In order to develop such CPUs, CAD and methodology progress is necessary. In verification area, for instance, much faster simulation/emulation techniques and robust formal verification will be necessary. In backend area, "interconnection centric" tools ranging over floor-plan, logic synthesis, place \& route, extraction and timing analysis will be necessary. As signal swing gets smaller and coupling capacitance among adjacent wires is increased, induced noise on a signal wire will become more serious problem. A CAD tool to estimate noise immunity will be necessary, too.

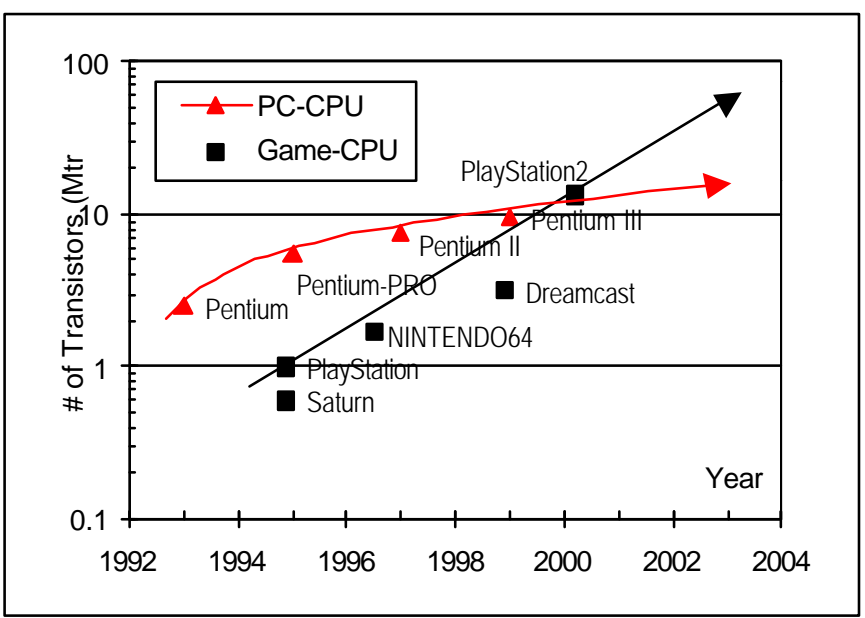

Fig.4 CPU Integration Trend

\section{Acknowledgements}

The authors would like to thank Ken Mabuchi, Itaru Yamazaki, Toshiyuki Sakamoto, Kennichi Mori, Ichiro Nagashima, Teiji Yutaka for their contribution in verification and Masataka Matsui for leadership of timing improvement.

\section{References}

[1] Ken Kutaragi, et al., "A Microprocessor with a 128b CPU, 10 Floating-Point MACs, 4 Floating-Point Dividers, and an MPEG2 Decoder," IEEE ISSCC Digest of Technical Papers, Vol.42, pp.256-257, 1999

[2] F. Michael Raam, et al., "A High Bandwidth Superscalar Microprocessor for Multimedia Applications," IEEE ISSCC Digest of Technical Papers, Vol.42, pp.258-259, 1999.

[3] T. Kamei, et al., "300MHz design methodology of VU for Emotion Synthesis," Proc. ASP-DAC, to be appeared., 2000.

[4] N. Kojima, et al., "Repeater Insertion Method and its application to a $300 \mathrm{MHz}$ 128-bit 2-way Superscaler Microprocessor," Proc. ASP-DAC, to be appeared., 2000.

[5] F. Ishihara, et al., "Clock Design of $300 \mathrm{MHz} 128$-bit 2-way Superscaler Microprocessor," Proc. ASP-DAC, to be appeared., 2000. 